\title{
Verification of Surface Conductance Model of Textile Materials
}

\author{
M. Neruda*1, L. Vojtech ${ }^{2}$ \\ ${ }^{1,2}$ Czech Technical University in Prague, FEE, \\ Dept. of Telecommunication Engineering Technicka 2, 16627 \\ Prague, Czech Republic \\ *marek.neruda@fel.cvut.cz
}

\begin{abstract}
Surface conductance is one of the main parameters which describes basic characteristics of specific textile materials. These textile materials are used, for example, in environments with higher requirements on hardware electrostatic protection during their production process. Published measurement standardized methods are followed by modeling methods which do not reach satisfactory results. This paper is therefore focused on the modeling of surface conductance of textile materials. The results show that the structure of textile materials can be seen as serial-parallel connections of resistors. A derivation of this model is described.
\end{abstract}

Keywords: derivation, surface conductance, textile material.

\section{RESUMEN}

La superficie de la conductancia se encuentra dentro de los parámetros principales de materiales textiles específicos y describe sus características básicas. Estos materiales se usan por ejemplo en situaciones donde se requiere una protección electroestática para el hardware durante el proceso de producción. Los métodos de medida estándares publicados siguen métodos modelados que no alcanzan resultados satisfactorios. Por lo tanto, este informe se enfoca en modelar la superficie de la conductancia de materiales textiles. Los resultados muestran que la estructura de los materiales textiles pueden considerarse como conexiones de resistencias serie-paralelo. Se describe, además, una derivación de este modelo.

\section{Introduction}

Surface conductance of textile materials is often caused by coating of the textile which is composed of non-conductive fibers. This type of textiles achieves high values of surface conductance in wide frequency band and is used, for example, in the construction of flexible antenna structures. Surface conductance modeling is generally based on the examination of conductance of upper metal layer of originally non-conductive textiles. The surface resistance of these textile materials is calculated as resistance between standardized electrodes [1]. It is equivalent to $1 /(t \cdot \sigma)$ [2], where $\sigma$ is the conductance of coating material on the textile surface with thickness $t$. Surface conductance modeling is generally performed by 3D software [3], which simulates a specific model. Present modeling methods are not sufficient in the case of requirements of exact modeling of textile

materials' surface conductance during their production. Simplified models mainly malfunction with textiles with low surface conductance which are produced from carbon, for example. Carbon fibers are characterized by electric conductivity in their whole cross section and, therefore, these textiles are classified as textile materials with conductive fibers. Resultant conductance of these fibers cannot be replaced by surface conductance of theoretical homogeneous sheets which is used at coating material modeling. The reason is seen in electric conductivity, which is an indispensable component of inhomogeneity between weft and warp. Presented models completely malfunction at requirement of modeling of textile materials with the ability to distribute electrostatic charge. The textiles are used for protection against undesirable effects of accumulated charges with the aid of 
electrostatic discharge (ESD). The reason of malfunctioning is due to the structure of textiles. Bigger distances in warp and weft form rather conductive grid than homogeneous structures. Standard statistic and geometric models malfunction similarly. These models are used for a description of electric characteristics of multicomponent materials [4].

The model of these electroconductive textile materials, produced from coated non-conductive fibers and conductive fibers, can be seen from the electrical point of view as finite grid of resistors. The fibers form the resultant structure of the textile. A close investigation of the structure shows, in a big magnification, it is a structure of crossed fibers which form regular shapes in general. In ideal case these shapes are squares or rectangles with sides composed of fibers and the air between them. Every side of square, special case of rectangle, represents one resistor. These resistors are connected together and form a finite grid. The paper describes the calculation of a regular square finite grid of resistors, which is verified by simulation software. Standardized measurement methods are used for valuation of length resistance [5].

The model and its calculation result from the application of textile materials with antistatic conductive fibers which are used in ESD applications for protection of electronic devices. The confirmation is performed with respect to dimensions of standardized probes [6] and common distance of conductive fibers in textile materials $[4,7]$.

The rest of the paper is organized as follows: Section 2 describes the derivation of a recently published formula of resistance of a regular resistor grid. Section 3 introduces a verification of an equipotential line presumption by simulation and calculation model. Finally, Section 4 presents the conclusions.

\section{Resistance of Grid of Resistors}

A calculation of the resultant resistance requires that the grid of resistors is connected to the electrical circuit. Poles of battery can be connected on both sides of the grid; however, it is better to consider standard EN 1149-1:2006 [1] or EN 613405-1 [2] which describe a test method for measurement of surface resistivity. A gauging fixture of the method is composed of coaxially ordered electrodes. The inner probe diameter is 30 $\mathrm{mm}$ and the outer one is $63 \mathrm{~mm}$ in [6]. A measured sample is put between them. This setting of measurement experiments leads to an idea to connect one pole of battery in the middle of the grid and the second one on the outer edge of the grid.

The first step of the calculation is to find and verify the formula, describing the rectangular structure of the textile material; therefore, the calculation focuses on rectangular shapes of inner and outer electrodes. The second step is specification of this formula for the circle shape.

Previous research shows it is possible to calculate the resultant resistance as [8]

$$
R=\frac{R^{\prime}}{8}+\frac{1}{4} \cdot \sum_{n=5}^{r} \frac{R^{\prime}}{n-1}+\frac{1}{4} \cdot \sum_{n=5}^{r} \frac{R^{\prime}}{n-3}, n \in\langle 5, r), r, \text { nareodd }
$$

where $R$ denotes the resultant resistance, $R^{\prime}$ represents a length resistance of fiber for square dimension, $r$ and $n$ are connected resistors $R^{\prime}$ in the horizontal direction.

Despite the resultant formula being published, the complete derivation is not described and the calculation is not verified by other methods. The first part of (1), $R^{\prime} / 8$, represents 8 parallel resistors connected between two poles of battery, or electrodes. It forms a shape depicted in Fig. 1.

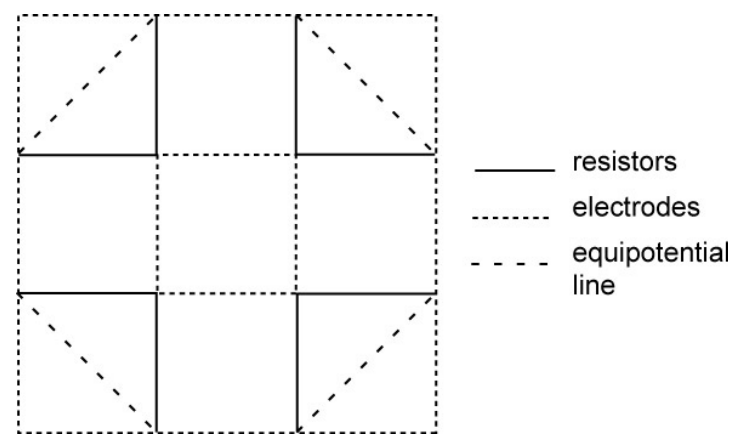

Figure 1. Grid of resistors $\left(n=3, R^{\prime}=8\right)$. 
The second part of (1) describes connected resistors between the electrodes, or poles of batteries. The calculation uses a presumption of connection of nodes with same potentials. Fig. 1 depicts the equipotential line for $n=3$. The solid line represents 8 resistors which are connected in parallel between two electrodes. For $n=5$, the second group of resistors in a row of calculation contains 16 resistors. This fact is also shown in Fig. 2. If $n$ is increased, other resistors are added into the grid. These resistors are only added as an addend of the current ones, but they are connected in parallel with each other. It means parallel-connected resistors are added up with other parallel connected resistors as connection in series. The reason is of course in the same potential. This presumption is fundamental for the correct derivation of the model and, therefore, the following sections focus on its confirmation.

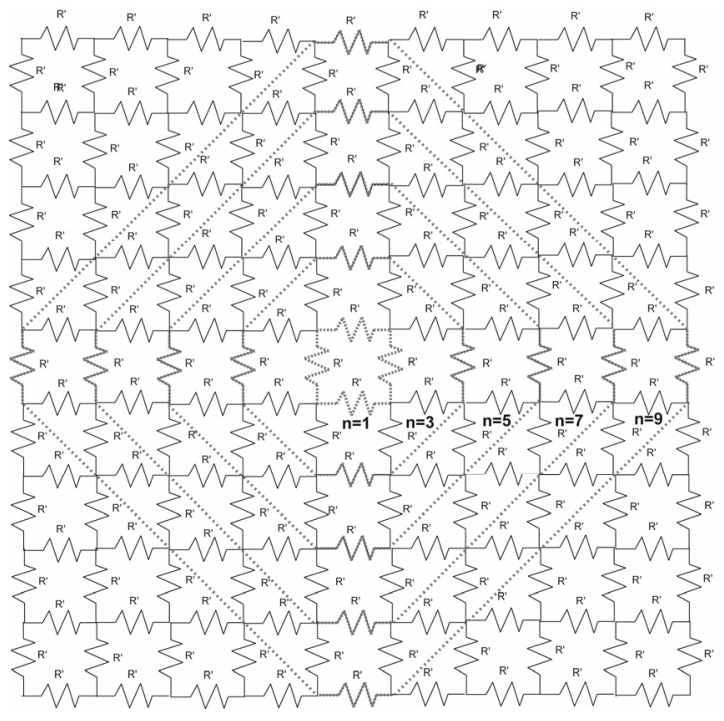

Figure 2. Presumption of points with the same potential of the textile material.

Considering $n=3, n=5, n=7$ and $n=9$, the resultant resistance is calculated as

$$
R=\frac{R^{\prime}}{8}+\frac{R^{\prime}}{16}+\frac{R^{\prime}}{24}+\frac{R^{\prime}}{32}
$$

where $R^{\prime} / 8$ is the 8 parallel-connected resistors for $n=3$ and, accordingly, $R^{\prime} / 16$ for $n=5, R^{\prime} / 24$ for $n=7$ and $R^{\prime} / 32$ for $n=9$.
Equation (2) is rewritten as

$$
R=\sum_{m=1}^{r} \frac{R^{\prime}}{8 \cdot m}
$$

where $m, r \in\{1,2,3,4\}$.

Equation (3) can be extended to increase $n$, and it leads to an extension of set of parameters $m, r$, therefore, $m, r \in\{\mathrm{N}\}$. The relation between parameter $n$ and $m$ can be calculated from:

$$
\begin{gathered}
m=1, n=3 \Rightarrow m=n-2, \\
m=2, n=5 \Rightarrow m=n-3, \\
m=3, n=7 \Rightarrow m=n-4, \text { etc. }
\end{gathered}
$$

The resultant formula of (4) can be expressed as

$$
m=n-(2+x)
$$

where $x \in\{0,1,2, .$.$\} .$

\section{Considering:}

$$
\begin{gathered}
x=0, n=3, \\
x=1, n=5, \\
x=2, n=7, \text { etc },
\end{gathered}
$$

The relation between parameters $\mathrm{x}$ and $\mathrm{n}$ is calculated as

$$
x=\frac{n+1}{2}-2
$$

If (7) is substituted into (3), the second part of (1) can be expressed. A value $m=1$ is eliminated because it is already expressed as the first part of (1).

$$
\begin{gathered}
R=\sum_{m=2}^{r} \frac{R^{\prime}}{8 \cdot m}=\sum_{n=5}^{r}\left(\frac{1}{8} \cdot \frac{R^{\prime}}{n-(2+x)}\right)=\frac{1}{8} \cdot \sum_{n=5}^{r} \frac{R^{\prime}}{n-\left(2+\frac{n+1}{2}-2\right)}= \\
=\frac{1}{8} \cdot \sum_{n=5}^{r} \frac{R^{\prime}}{\frac{2 n-n-1}{2}}=\frac{1}{4} \cdot \sum_{n=5}^{r} \frac{R^{\prime}}{n-1}
\end{gathered}
$$


The third part of (1) represents the rest of resistors which form the "corners" of Fig. 3. It is calculated from

$$
n=5 \Rightarrow R=\frac{R^{\prime}}{8}, n=7 \Rightarrow R=\frac{R^{\prime}}{8}+\frac{R^{\prime}}{16}, n=9=
$$

This dependence is used as

$$
R=\frac{1}{4} \cdot\left(\frac{R^{\prime}}{2}+\frac{R^{\prime}}{4}+\frac{R^{\prime}}{6}\right)=\frac{1}{4} \cdot\left(\frac{R^{\prime}}{5-3}+\frac{R^{\prime}}{7-3}+\frac{R^{\prime}}{9-3}\right)=\frac{1}{4} \cdot \sum_{n=5}^{r} \frac{R^{\prime}}{n-3}
$$

\section{Rerification of Equipotential Line Validity}

The confirmation is performed with respect to ESD applications for protection of electronic devices. It is possible to use the probes with diameters 30 $\mathrm{mm}$ and $63 \mathrm{~mm}$ [6], which are produced according to EN 61340-5-1 [2]. Common distance of antistatic conductive fibers in weft in these textile materials is approximately $10 \mathrm{~mm}[4,7]$. Considering a square with dimensions $10 \times 10$ $\mathrm{mm}$, inner diameter includes 3 squares and outer 5 squares. Seven squares are not considered because all resistors between $n=5$ and $n=7$ are not included. Therefore, $n=5$ is considered as a sufficient value for the applications, which require protection against undesirable effects of ESD.

Equation (1) is valid if every group of parallel connected resistors has one connection end in one potential level and the second connection end in other potential level. Then the resultant resistance is calculated as a pure addition. A lot of programs exist which enable to simulate characteristics of electrical circuits. It is possible to use, for example, a program called Oregano, GNOME application for schematic capture and printing of electrical circuits [9]. Fig. 3 depicts a simulated electrical circuit. It also shows a placement of voltage probes of the same potential levels. The results are depicted in Fig. 4. It shows only one line of two probes because the lines are covered with one another. The values of voltage are, therefore, the same.
This simulation confirms the presumption of potential levels for $n=5$.

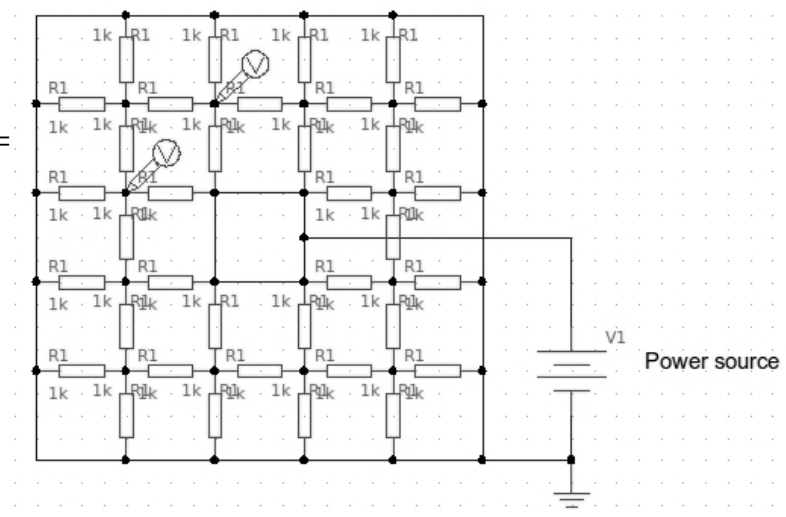

Figure 3. Simulated electric circuit for $n=5$.

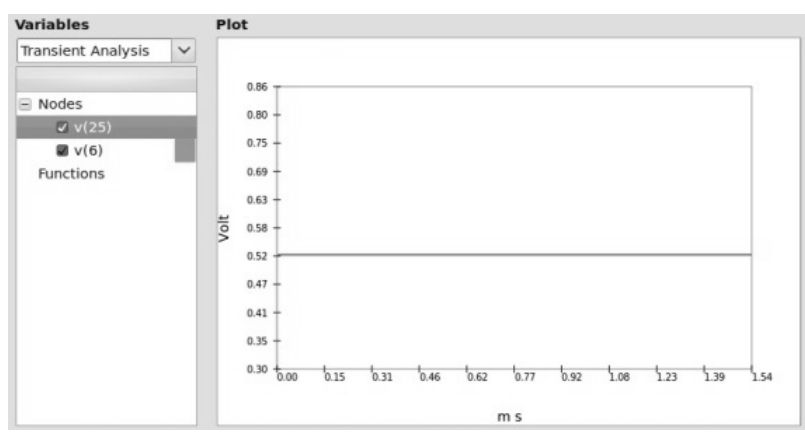

Figure 4. Voltage in chosen points of electrical circuit for $n=5$.

Equation (1) is also interesting from another point of view. Equipotential lines cause some resistors to be equal to zero in the resultant resistance. These resistors are connected by their both ends to the same potential level. They are placed on horizontal and vertical lines which cross the inner electrode. Simulation results show this presumption is confirmed as well because the same voltage value is measured on both ends of these resistors; however, this presumption can also be confirmed or disconfirmed by a calculation of the resultant resistance with the aid of electrical circuit laws. Basic rules related to the counting of parallel- or serial-connected resistors and transformation delta-star, star-delta are used in the simplification of circuits. These electrical laws are used in Fig. 5, Fig. 6 and Fig. 7 for $n=5$. They even depict values of individual resistors, where $R 1$ in the figures is equal to $R^{\prime}$ in the calculation. The 
value of the resultant resistance is shown in Fig. 7 , scheme f), it is $5 / 24 R^{\prime}$.

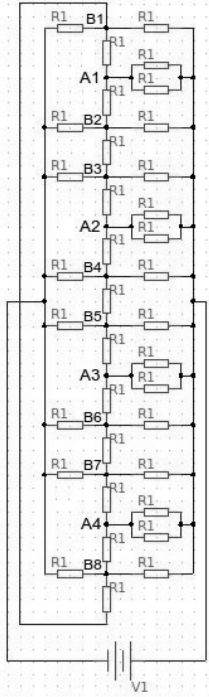

a) b)

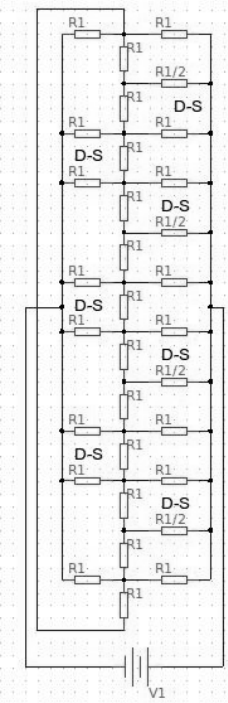

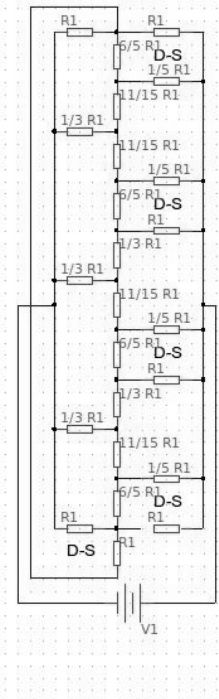

c)
Figure 5. Redrawn electrical circuit and its modification by Delta-star (D-S) transformation $(n=5)$.

If an electric circuit in Fig. 6 a) and presumption of equipotential lines are considered, nodes marked A1-A4 and nodes B1-B8 have the same potential and can be connected or disconnected as necessary. It leads to elimination of resistors placed between nodes B1-B8, B2-B3, B4-B5 and B6-B7 which are connected by their both ends to the same potential level, Fig. 8. Resultant resistance is then calculated as (symbol \| indicates parallel connected resistors):

This result confirms the presumption of equipotential lines for $n=5$.

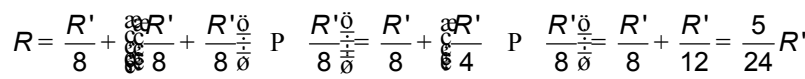

Considering (1), the result is

$$
R=\frac{R^{\prime}}{8}+\frac{1}{4} \cdot \frac{R^{\prime}}{4}+\frac{1}{4} \cdot \frac{R^{\prime}}{2}=\frac{2+1+2}{16} \cdot R^{\prime}=\frac{5}{16} \cdot R^{\prime}
$$

This formula has to be therefore specified. The difference is caused by the serial-parallel connection of resistors placed in the "corner" of the resistance grid. It is not simple addition of resistors. The specified derivation is obtained from the connection, Fig. 9.
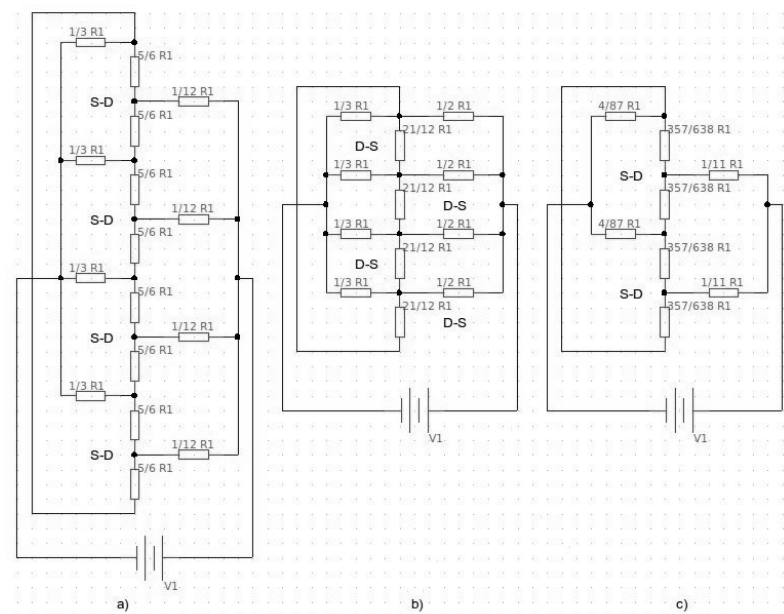

Figure 6. Modified electrical circuit by Delta-star (D-S) and Star-delta (S-D) transformation $(n=5)$.
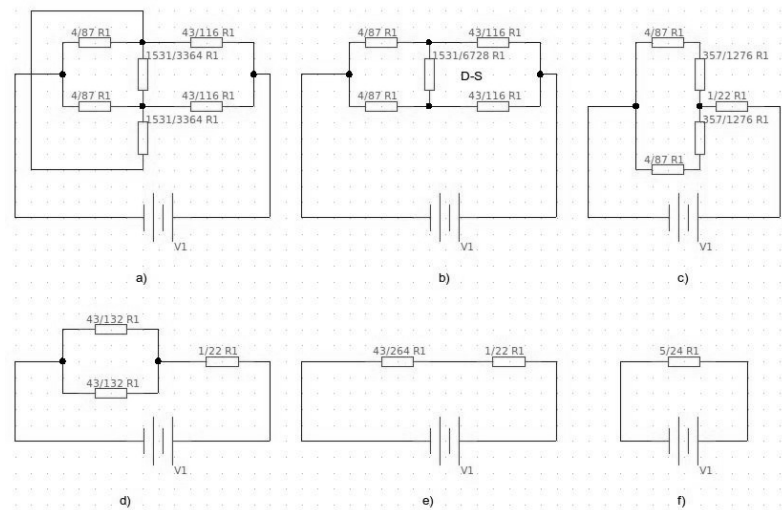

Figure 7. Value of resultant resistance of electric circuit is marked f) $(n=5)$.

This formula has to be therefore specified. The difference is caused by the serial-parallel connection of resistors placed in the "corner" of the resistance grid. It is not simple addition of resistors. The specified derivation is obtained from the connection, Fig. 9. 


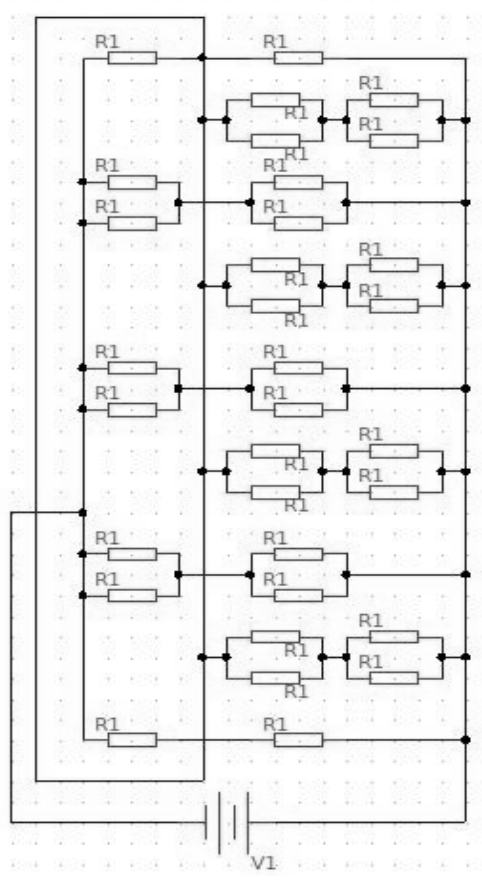

Figure 8. Simplified electrical circuit by eliminating specific resistors.

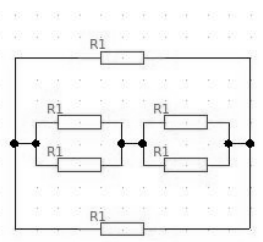

$n=5$

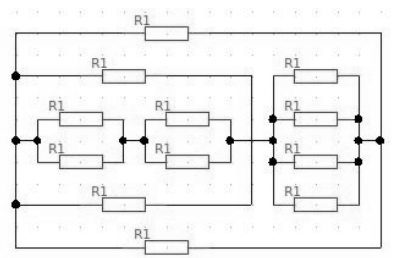

$\mathrm{n}=7$

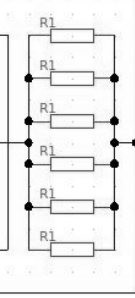

$n=9$

Figure 9. Series parallel connection of resistors in one "corner" of the grid.

Resistance of all four corners of the grid for $n=5$, $n=7$ and $n=9$ is expressed as

$$
\begin{gathered}
n=5 \Rightarrow R_{c}=\frac{R^{\prime}}{8}+\left[\left(\frac{R^{\prime}}{8}+\frac{R^{\prime}}{8}\right) \mathrm{P} \frac{R^{\prime}}{8}\right] \\
n=7 \Rightarrow R_{c}=\frac{R^{\prime}}{8}+\left\{\left[\left(\frac{R^{\prime}}{8}+\frac{R^{\prime}}{8}\right) \mathrm{P} \frac{R^{\prime}}{8}\right]+\frac{R^{\prime}}{16}\right\} \mathrm{P} \frac{R^{\prime}}{8} \\
n=9 \Rightarrow R_{c}=\frac{R^{\prime}}{8}+\left[\left(\left\{\left[\left(\frac{R^{\prime}}{8}+\frac{R^{\prime}}{8}\right) \mathrm{P} \frac{R^{\prime}}{8}\right]+\frac{R^{\prime}}{16}\right\} \mathrm{P} \frac{R^{\prime}}{8}\right)+\frac{R^{\prime}}{24}\right] \mathrm{P} \frac{R^{\prime}}{8}
\end{gathered}
$$

This dependence is generally rewritten as

$$
R_{c}=\left(R_{n-2}^{*}+\frac{R^{\prime}}{4(n-3)}\right) \mathrm{P} \frac{R^{\prime}}{8}, R_{5}^{*}=\left(\frac{R^{\prime}}{8}+\frac{R^{\prime}}{8}\right) \mathrm{P} \frac{R^{\prime}}{8}
$$

This improvement of (1) describes resistance of the "corners" of the grid; however, it includes also resistors placed on the equipotential line which crosses the outer electrode, Fig. 2. These resistors are eliminated in the second part of (2). The specified formula is expressed as:

$R=\frac{R^{\prime}}{8}+\frac{1}{4} \sum_{n=5}^{r-2} \frac{R^{\prime}}{n-1}+\left(R_{n-2}^{*}+\frac{R^{\prime}}{4(n-3)}\right), n \in\langle 5, r), r$, nare odd,$R_{5}^{*}=\left(\frac{R^{\prime}}{8}+\frac{R^{\prime}}{8}\right) \mathrm{P} \frac{R^{\prime}}{8}$

According to the specified formula (15), the resultant resistance for $n=5$ can be calculated for the verification of the electrical circuit depicted in Fig. 5 as

$R=\frac{R^{\prime}}{8}+0+\left[\left(\frac{R^{\prime}}{8}+\frac{R^{\prime}}{8}\right) \mathrm{P} \frac{R^{\prime}}{8}\right]=\frac{R^{\prime}}{8}+\left(\frac{R^{\prime}}{4} \mathrm{P} \frac{R^{\prime}}{8}\right)=\frac{R^{\prime}}{8}+\frac{R^{\prime}}{12}=\frac{5}{24} R^{\prime}$

The result (16) confirms that the specified formula for rectangular shapes of electrodes is valid. It also verifies the presumption of equipotential lines for $n=5$. Considering the dimensions of the inner rectangular electrode, it is possible to subtract $n$ resistors $\mathrm{R}^{\prime}$ in the horizontal and vertical direction of the grid. 


\section{Conclusions}

This paper focuses on the description of the surface conductance model which is applicable to antistatic textile materials used in ESD applications for the protection of electronic devices. A presumption of equipotential lines is introduced and verified by a simulation software and by the calculation of the resistance of the electric circuit. The results show the presented formula for resistance calculation is valid for this application. Future work will consider shorter dimensions of conductive fibers which will decrease the intensity of the electric field and charge of electricity. It will enable even integrated circuits to make contact with this textile material. The resulting goal of this work is a model which considers circle shapes of electrodes of surface conductance measurement standard. This will enable to model the textile before its production.

\section{References}

[1] Standard BS EN 1149-1:2006: Protective clothing. Electrostatic properties. Test method for measurement of surface resistivity, Available at: http://shop.bsigroup.com/en/

ProductDetail/?pid $=000000000030112375$

[2] Lilja, J. \& Salonen, P., On the modeling of conductive textile materials for SoftWearAntennas, Antennas and Propagation Society International Symposium, 2009, pp.1-4, Charleston, South Carolina, USA, June.

[3] Li, L., AU, W. M., Wan, K. M., Wan S. H., Chung W. Y., Wong K. S., A Resistive Network Model for Conductive Knitting Stitches, Textile Research Journal, Vol. 80, No. 10, November, 2009, p. 935-947.

[4] Marsalkova, M., Electric behavior of Textiles with Antistatic Properties and Methods of Their Evaluation, PhD. Thesis, Technical University of Liberec, Czech Republic, 2008.

[5] Safarova, V. \& Gregr, J., Electrical Conductivity Measurement of Fibers and Yarns, 7th International Conference TEXSCI, 2010, Liberec, Czech republic, September.

[6] Concentric Ring Probes, Vermason, The Specialists in ESD Protection [online], 2012 [cit. 2012-01-26], Available at: http://www.vermason.co.uk/.

FullCatalog/TestEquipment/SurfaceResistanceTesters/C oncentricRingProbes/
[7] Novak, M., The Model of Surface Electrical Conductivity of Special Fabrics, Master Thesis, CTU in Prague, Czech republic, 2011.

[8] Neruda, M. \& Vojtech, L., Surface Conductance of Textile Materials Modeling, Knowledge in Telecommunication Technologies and Optics, December, 2010, p. 171-175.

[9] Ubuntu [online]. 2011 [cit. 2011-03-24]. Package: oregano (0.69.1-1ubuntu1.10.04.1) [universe]. Available: http://packages.ubuntu.com/lucidupdates/gnome/oregano>. 